\title{
TRIPLE POINTS AND SINGULARITIES OF PROJECTIONS OF SMOOTHLY IMMERSED SURFACES
}

\author{
THOMAS BANCHOFF
}

\begin{abstract}
For a transversal smooth immersion of a closed 2-dimensional surface into Euclidean 3-space, the number of triple points is congruent modulo 2 to the Euler characteristic. The approach of this paper includes an introduction to normal Euler classes of smoothly immersed manifolds by means of singularities of projections.
\end{abstract}

In this note we prove that for a (transversal) smooth immersion of a closed 2-dimensional surface into Euclidean 3-space, the number of triple points is congruent modulo 2 to the Euler characteristic. The approach used here includes an introduction to the normal Euler class of a smoothly immersed manifold and is related to the theory of Stiefel-Whitney classes in terms of singularities of projections as developed in [2].

The main result of this paper is correct also for manifolds which are not smooth, and such a proof has been carried out using surgery techniques in [3].

1. Singularities of projections and the Euler characteristic. Let $f: M^{2} \rightarrow \mathbf{R}^{3}$ be a smooth immersion of a closed 2-dimensional surface into real 3-dimensional space with a coordinate system $(x, y, z)$. Let $\pi_{z}: \mathbf{R}^{3} \rightarrow$ $\mathbf{R}(z)$ denote the orthogonal projection into the $z$-axis and let $\mathcal{S}_{z}(f)$ be the set of critical points of $\pi_{z} \circ f: M^{2} \rightarrow \mathbf{R}(z)$, i.e. the set of points where the tangent plane to $f(M)$ is orthogonal to the $z$-axis. Let $\pi_{x z}: \mathbf{R}^{3} \rightarrow \mathbf{R}(x, z)$ denote the orthogonal projection into the $x z$-plane, and let $\mathcal{S}_{x z}(f)$ be the set of singularities of $\pi_{x z} \circ f: M^{2} \rightarrow \mathbf{R}(x, z)$, i.e. the set of points where the tangent plane to $f(M)$ is orthogonal to the $x z$-plane.

We assume that the coordinate system in 3-space is so chosen that the set $\mathcal{S}_{z}(f)$ consists of a finite number of points, consisting of $m_{0}\left(\pi_{z} \circ f\right)$ local minima, $m_{1}\left(\pi_{z} \circ f\right)$ nondegenerate saddle points, and $m_{2}\left(\pi_{z} \circ f\right)$ local maxima.

Received by the editors November 15, 1973. AMS (MOS) subject classifications (1970). Primary 57D45, 57D20, 57D35. Key words and phrases. Triple points, singularities of projections, immersions, Euler characteristic. 
If $A$ is a finite set, let $N(A)$ denote the number of points of $A$.

Proposition 1. For an immersion satisfying the above conditions, $N\left(\mathcal{S}_{z}(f)\right) \equiv \chi\left(M^{2}\right)(\bmod 2)$.

Proof. For a function $\pi_{z} \circ f$ satisfying the above conditions, the Euler characteristic $\chi\left(M^{2}\right)$ can be expressed as

$$
\chi\left(M^{2}\right)=m_{0}\left(\pi_{2} \circ f\right)-m_{1}\left(\pi_{z} \circ f\right)+m_{2}\left(\pi_{z} \circ f\right)
$$

(for an elementary treatment of this result see [1]). But then

$$
\begin{aligned}
N\left(\mathcal{S}_{z}(f)\right) & =m_{0}\left(\pi_{z} \circ f\right)+m_{1}\left(\pi_{z} \circ f\right)+m_{2}\left(\pi_{z} \circ f\right) \\
& =\chi\left(M^{2}\right)+2 m_{1}\left(\pi_{z} \circ f\right) \equiv \chi\left(M^{2}\right)(\bmod 2) .
\end{aligned}
$$

Proposition 2. $N\left(\mathcal{S}_{x z}(f) \cap \mathcal{S}_{y z}(f)\right)=N\left(\mathcal{S}_{z}(f)\right)$.

Proof. This follows from the observation that the tangent plane to $f(M)$ at $f(p)$ is orthogonal to the $z$-axis if and only if it is orthogonal to both the $x z$ - and $y z$-planes.

Remark. If $M^{2}$ is a domain in the $x y$-plane and if $f: M^{2} \rightarrow \mathbf{R}^{3}$ is given by a graph of a differentiable function $g(x, y)$ so $f(x, y)=(x, y, g(x, y))$, then $\mathcal{S}_{x z}(f)$ consists of all $(x, y)$ in $M$ such that $(\partial g / \partial y)(x, y)=0$ and $\mathcal{S}_{y z}(f)=\{(x, y)$ in $M \mid(\partial g / \partial x)(x, y)=0\}$. The intersection $\mathcal{S}_{x z}(f) \cap \mathcal{S}_{y z}(f)=$ $\{(x, y)$ in $M \mid(\partial g / \partial x)(x, y)=0=(\partial g / \partial y)(x, y)\}$ and these are the critical points of the height function in the $z$ direction.

2. The double locus and the fold set. Let $I(f)$ be the set of double points of $f$, i.e. $I(f)=\{p$ in $M \mid f(q)=f(p)$ for exactly one $q \neq p$ in $M\}$. We assume that $f$ is transversal so that if $p \neq q$ but $f(p)=f(q)$, then the tangent planes to $f(M)$ at $f(p)$ and $f(q)$ intersect in a line. We assume further that if $f\left(p_{1}\right)=f\left(p_{2}\right)=f\left(p_{3}\right)$ for distinct points $p_{1}, p_{2}, p_{3}$, then the tangent planes to $f(M)$ at $f\left(p_{1}\right), f\left(p_{2}\right)$ and $f\left(p_{3}\right)$ intersect at a single point. We assume moreover that there is no point of $\mathbf{R}^{3}$ which is the image of more than three points of $M$.

We assume as well that the coordinate system of $\mathbf{R}^{3}$ has been chosen so that $I(f) \cap \mathcal{S}_{z}(f)=\varnothing$ and so that $I(f)$ is transversal to both $\mathcal{S}_{x z}(f)$ and $\delta_{y z}(f)$. This, in particular, means that $I(f) \cap \mathcal{S}_{x z}(f)$ consists of a finite number of points, $N\left(I(f) \cap \mathcal{S}_{x z}(f)\right)$, and that at each point $p$ of $\mathcal{S}_{x z}(f) \cap I(f)$, there is exactly one $q \neq p$ in $M$ with $f(p)=f(q)$, and the tangent plane to $f(M)$ at $f(q)$ is not orthogonal to the $x z$-plane. 
Lemma. $\mathcal{S}_{y z}(f)$ and $I(f)$ together form the boundary of a 2-dimensional region on $M$.

Proof. From any point $f(p)$ we may consider the open ray in the direction of the positive $x$-axis and see how many times the ray meets the image of $f(M)$. We say that such a ray intersects $f(M)$ at $f(q)$ if the ray contains $f(q)$ and if the tangent plane to $f(M)$ at $f(q)$ does not contain the ray. Let $\mathcal{O}(f, y z)=\{p$ in $M \mid$ the open ray from $f(p)$ in the $x$-direction intersects $f(M)$ at $f(q)$ for an odd number of distinct points $q_{i}$ of $\left.M\right\}$. The boundary curves of this region include the points of $\mathcal{S}_{y z}(f)$ and the points of $I(f)$, and any point not in the closure of these two sets has a neighborhood which either is contained in $\mathcal{O}(f, y z)$ or is disjoint from this region. Thus $d \mathcal{O}(f, y z)=$ $I(f) \cup \mathcal{S}_{y z}(f)$.

As a corollary of this lemma, we have

Proposition 3. $N\left(\mathcal{S}_{y z}(f) \cap \mathcal{S}_{x z}(f)\right) \equiv N\left(I(f) \cap \mathcal{S}_{x z}(f)\right)(\bmod 2)$.

Proof. Each curve of $\mathcal{S}_{x z}(f)$ alternately enters and leaves the region $\mathcal{O}(f, y z)$ and ends up where it started, so $\mathcal{S}_{x z}(f)$ meets the boundary $d \mathcal{O}(f, y z)$ an even number of times. Thus $0 \equiv N\left(\mathcal{S}_{x z}(f) \cap d \mathcal{O}(f, y z)\right) \equiv$ $N\left(\mathcal{S}_{x z}(f) \cap I(f)\right)+N\left(\mathcal{S}_{x z}(f) \cap \mathcal{S}_{y z}(f)\right)(\bmod 2)$.

Remark. The above argument shows that for any sufficiently general orthogonal projection $\pi \circ f: M^{2} \rightarrow E^{2}$ into a plane, the singularity set is a collection of curves which determines a homology class independent of the projection, since each is homologous to $I(f)$. This homology class is the first normal Whitney class of $f$.

3. Triple points. The image $D(f)$ of $I(f)$ consists of a collection of closed curves in $\mathbf{R}^{3}$ and a collection of curve segments with both endpoints in the set of triple points $T(f)=\left\{s\right.$ in $\mathbf{R}^{3} \mid s=f\left(p_{1}\right)=f\left(p_{2}\right)=f\left(p_{3}\right)$ for distinct points $p_{1}, p_{2}, p_{3}$ of $\left.M\right\}$.

Proposition 4. $N\left(\mathcal{S}_{x z}(f) \cap I(f)\right) \equiv N(T(f))(\bmod 2)$.

Proof. Let $O D(f, x z)=\{s$ in $D(f) \mid$ the open ray from $s$ in the $y$ direction intersects $f(M)$ at an odd number of points $\left.f\left(q_{i}\right)\right\}$. The set $O D\left(f_{1} x z\right)$ is a collection of closed curves and curve segments with endpoints either in $T(f)$ or in the image of the set $\mathcal{S}_{x z}(f) \cap I(f)$.

Note that even though each point in $D(f)$ has two pre-images in $M$, no two points of $\mathcal{S}_{x z}(f) \cap I(f)$ have the same image, so $N\left(\mathcal{S}_{x z}(f) \cap I(f)\right)=$ $N\left(f\left(\mathcal{S}_{x z}(f) \cap I(f)\right)\right)$. Each of these image points lies in the boundary 


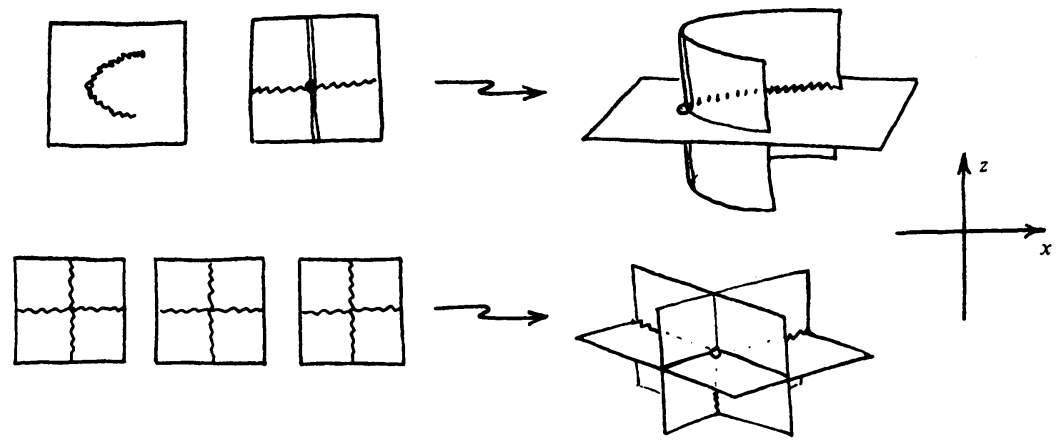

$d(\Theta D(f, x z))$, and each triple point lies in the boundary of exactly three of the segments of $O D(f, x z)$. Therefore

$$
\begin{aligned}
N\left(f \left(\mathfrak{S}_{x z}(f)\right.\right. & \cap l(f))+3 N(T(f))) \\
= & 2 N(\text { curve segments in } O D(f, x z)) \equiv 0(\bmod 2) .
\end{aligned}
$$

It follows that $N\left(\mathcal{S}_{x \boldsymbol{z}}(f) \cap I(f)\right) \equiv N(T(f))(\bmod 2)$.

Remark 1. The foregoing argument can be summarized by saying that the Euler class of the tangent bundle of $M$ in $H^{2}\left(M^{2} ; Z_{2}\right)$ is equal to the cup product square of the first normal Whitney cohomology class, $\bar{W}^{1}$, and this is given by the Poincaré dual of the intersection product of the 1-dimensional fold homology class which is the Poincare dual of $\bar{W}^{1}$, and the intersection product is homologous to three times the triple point set.

Remark 2. For smoothly immersed surfaces in $\mathbf{R}^{3}$, the Whitney duality theorem $1=\left(1+\bar{w}_{1}\right) \cup\left(1+w_{1}+w_{2}\right)$ implies that $w_{1}=\bar{w}_{1}$ and $w_{1} \cup w_{1}=$ $w_{2}$, so the method of $\oint_{1}$ may be considered as a geometric interpretation of this result. More generally for a smoothly immersed hypersurface $M^{n-1}$ in $\mathbf{R}^{n}$, the Whitney duality theorem $1=\left(1+\bar{w}_{1}\right) \cup\left(1+w_{1}+\cdots+w_{n-1}\right)$ implies that $w_{1}=\bar{w}_{1}$ and $w_{i}=\left(w_{1}\right)^{i}$ for any $i=1,2, \cdots, n-1$. Again the method of $\S_{1}$ provides a geometric interpretation of this result since the intersection of $i$ fold $(n-1)$-chains transversally situated will be an $(n-i-1)$ dimensional chain which is the singularity chain for a projection to an $(n-1)$ dimensional linear subspace and the homology class of such a chain is the Poincaré dual of the $i$ th Stiefel-Whitney cohomology class of $M^{n-1}$ (compare [2]). The author would like to thank Glen Bredon for his comments leading to this remark.

Remark 3. The author would like to thank James White for helpful conversations during this project and in particular for discussions of his manu- 
script [5]. The result of this paper is related to that of Lashof and Smale [4] which uses techniques of much greater sophistication. (The authors have communicated the fact that this paper contains some errors.)

\section{BIBLIOGRAPHY}

1. T. F. Banchoff, Critical points and curvature for embedded polyhedral surfaces, Amer. Math. Monthly 77 (1970), 475-485. MR 41 \#4444.

2. - Stiefel Whitney homology classes and singularities of projections for polyhedral manifolds, Proc. Sympos. Pure Math., vol. 27, Amer. Math. Soc., Providence, R. I., 1975 (to appear).

3. - Triple points and surgery of immersed surfaces, Proc. Amer. Math. Soc. 46 (1974), 407-413.

4. R. K. Lashof and S. Smale, Self-intersections of immersed manifolds, J. Math. Mech. 8 (1959), 143-157. MR 21 \#332.

5. J. White, Twist invariants and Pontrjagin classes of immersed manifolds, Proc. Sympos. Pure Math., vol. 27, Amer. Math. Soc., Providence, R. I., 1975 (to appear).

\section{DEPARTMENT OF MATHEMATICS, UNIVERSITY OF CALIFORNIA, LOS ANGELES, CALIFORNIA 90024}

Current address: Department of Mathematics, Brown University, Providence, Rhode Island 02912 